\title{
Composição florística e estrutura da vegetação da savana de Miombo em dois ambientes distintos no Distrito de Sanga, Norte de Moçambique
}

O presente estudo teve como objetivo avaliar a composição e a estrutura da vegetação em dois ambientes de Miombo, sendo o primeiro denominado de Ambiente I (trecho de mata de Miombo), o segundo de Ambiente II (área abandonada após agricultura). Foram estabelecidas quinze parcelas de $20 \mathrm{~m} \times 50 \mathrm{~m}$ em cada ambiente. Todos os indivíduos lenhosos e em regeneração com DAP $>=5 \mathrm{~cm}$ foram medidos e identificados. A suficiência amostral foi avaliada com base nas curvas de rarefação. O padrão de variação florístico entre os ambientes avaliado através do Escalonamento Multidimensional não Métrico (NMDS). No Ambiente I, 40 espécies distribuídas em 21 famílias foram registradas enquanto que no ambiente II constatou-se a ocorrência de 19 espécies pertencentes a 10 famílias. Os parâmetros florísticos e fitossociológicos avaliados foram densidade, dominância, frequência e índice de valor de importância (IVI). Em ordem decrescente, Brachystegia spiciformis e Julbernardia globiflora apresentaram maiores IVI no Ambiente I. Uapaca kirkiana e Diplorhynchus condylocarpon detiveram os maiores valores para tal índice no Ambiente II. As espécies dominantes no Ambiente II são as comumente encontradas nesses ambientes e são capazes de crescer em áreas antropizadas e mostram uma capacidade de recuperação da vegetação do Miombo contra distúrbios antrópicos.

Palavras-chave: Sucessão ecológica; Estrutura da vegetação; Diversidade de espécies.

\section{Floristic composition and structure of Miombo savanna vegetation in two distinct environments in Sanga District, Northern Mozambique}

\begin{abstract}
The present study aimed to evaluate the composition and structure of the vegetation in two environments of Miombo, being the first denominated Site I (Miombo forest stretch) and Site II (abandoned area after agriculture). Fifteen plots of $20 \mathrm{~m} \times 50 \mathrm{~m}$ were established in each Site. All woody and regenerating individuals with $\mathrm{DBH}>=5 \mathrm{~cm}$ were measured and identified. Sampling adequacy was evaluated based on the rarefaction curves. The floristic variation pattern among the Sites evaluated through Non-Metric Multidimensional Scaling (NMDS). 40 species distributed in 21 families were registered, while in Site II 2, 19 species belonging to 10 families were found. The floristic and phytosociological parameters evaluated were density, dominance, frequency and importance value index (IVI). In descending order, Brachystegia spiciformis and Julbernardia globiflora presented the highest IVI in the Site I. Uapaca kirkiana and Diplorhynchus condylocarpon had the highest values for this index in Site II. The dominant species in Site II are those commonly found in these environments and are able to grow in anthropic areas and show a resilience recovery capacity of Miombo woodland.
\end{abstract}

Keywords: Ecological succession; Vegetation structure; Species diversity.

Topic: Uso Sustentável da Biodiversidade

Reviewed anonymously in the process of blind peer.

Merlindo Jacinto Manjate (i⿱

Universidade Lúrio, Moçambique

http://lattes.cnpq.br/6067118027738336

http://orcid.org/0000-0001-9574-9687

merlindomanjate@unilurio.ac.mz

Jonas Zefanias Massuque (iD

Universidade Lúrio, Moçambique

http://lattes.cnpq.br/7934340314867218

http://orcid.org/0000-0001-6162-0287

massuquejonas@gmail.com
Received: 02/12/2018

Approved: 26/01/2019
Referencing this:

MANJATE, M. J.; MASSUQUE, J. Z.. Composição florística e estrutura da vegetação da savana de Miombo em dois ambientes distintos no Distrito de Sanga, Norte de Moçambique. Revista Ibero Americana de Ciências Ambientais, v.10, n.1, p.77-89, 2019. DOI: http://doi.org/10.6008/CBPC2179-6858.2019.001.0007 


\section{INTRODUÇÃO}

O miombo é uma formação de savana florestal decídua que ocupa a maior extensão da África, cobrindo 2,7 milhões de $\mathrm{km}^{2}$ da sua porção sul, central e oriental (RIBEIRO et al., 2008). Apesar da sua importância ecológica e econômica, está ameaçado pelo desmatamento e a degradação do solo, sofrendo com elevada perda de biodiversidade (SILESHI et al., 2007). Estas ações humanas vêm causando uma drástica mudança na composição e estrutura das comunidades biológicas do Miombo, tornando os seus recursos cada vez mais modificados (BACKÉUS et al., 2006).

Dentre as causas da perda e alteração do Miombo estão a retirada da vegetação para a produção de carvão vegetal e a prática de corte e queima para a agricultura, que são os remotos e mais importantes tipos de uso da terra que afetaram essa ecoregião (SYAMPUNGANI et al., 2015). Em geral, a agricultura itinerante é o problema mais grave do uso de terra e o principal motor de desmatamento das áreas tropicais da África (KARTHIK et al., 2009). Em Moçambique as florestas têm sofrido impactos significativos devido a uma combinação de diversos fatores dentre os quais pode se destacar, o corte ilegal e desregrado de espécies madeireiras, a exploração de espécies para produção de carvão vegetal, a prática de agricultura itinerante, a frequência das queimadas descontroladas, a urbanização e as mudanças climáticas (MOURANA et al., 2007).

Com base nos dados do último inventário florestal nacional realizado a cada dez anos, a taxa de desmatamento em Moçambique foi estabelecida em 0,58\%, correspondente a uma perda florestal anual de 219 mil hectares (MARZOLI, 2007). Dentre as províncias moçambicanas, a de Niassa possui a menor densidade demográfica ( 1,5 milhão de pessoas), estando localizada na região norte do país. A maior parte da população do Niassa vive nas áreas rurais e é dependente da agricultura tradicional e de outros recursos naturais para sua subsistência (NHAMTUMBO et al., 2013).

No entanto, pouco ainda se sabe sobre a composição e estrutura da vegetação da Savana do Miombo em áreas abandonadas após agricultura em Moçambique, em especial no distrito de Sanga e no Posto Administrativo de Unango, o que dificulta quaisquer abordagens de maneio voltadas para o uso sustentável dos recursos florestais na região. Para que os projetos de conservação possam ser executados, é necessário que sejam conhecidos os ecossistemas onde se vai atuar, suas limitações e sua capacidade de recuperação e para tal se deve conhecer a composição florística e faunística, tanto em termos qualitativos como quantitativos, bem como as interdependências entre seus componentes (MARACAJÁ et al., 2003).

Neste trabalho, estudou-se a composição florística do estrato arbóreo e o comportamento da estrutura fitossociológica de dois ambientes de Miombo submetidos a dois níveis de antropismo: (uma área abandonada após agricultura e uma área não antropizada denominada trecho de mata do Miombo) visando obter informações para futuras ações de conservação ambiental. 


\section{METODOLOGIA}

\section{Área de estudo}

O estudo foi desenvolvido no posto Administrativo de Unango, no distrito de Sanga, província de Niassa (Figura 1). Esta região dista em $12 \mathrm{~km}$ da sede distrital Malulu, fazendo fronteira a norte com o Posto Administrativo de Macaloge, a Sul com o Distrito de Lichinga, a Este com o Distrito de Muembe através do rio Lucheringo e a oeste com o Posto Administrativo de Lussimbesse.

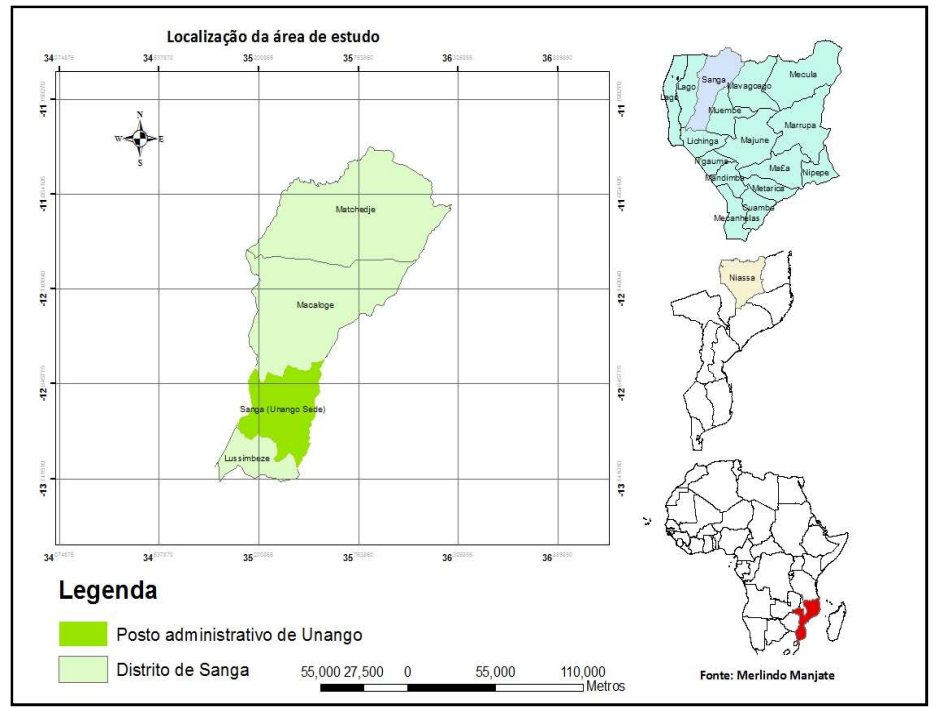

Figura 1: Localização geográfica do Posto Administrativo de Unango em relação à Moçambique e à África.

A agricultura é a atividade econômica dominante no distrito de Sanga, embora seja de subsistência, com a prática de corte e queima e com baixa produtividade (MAE, 2005). Este tipo de agricultura é praticada em pequenas parcelas de aproximadamente 2,5 ha, designadas de 'machambas' (LANDRY et al., 2011). Cada agregado familiar possui mais que uma machamba e, normalmente, de culturas diferentes, sendo as principais culturas cultivadas o milho, amendoim, feijão e batata.

Segundo o MAE (2005), o clima da região está sob influência da Zona de Convergência Intertropical (ZCIT), que origina duas estações bem definidas. A precipitação média anual oscila entre 1.000 a 1.200 mm no extremo norte, ao longo dos rios Rovuma e Lucheringo, chegando a atingir os máximos de $2.000 \mathrm{~mm}$ nas zonas mais altas da cordilheira. Os valores médios das temperaturas durante a estação quente e húmida são de 20 e $23^{\circ} \mathrm{C}$ na zona planáltica e na Cordilheira de Sanga os valores de temperatura tendem a aumentar para 23 a $26^{\circ} \mathrm{C}$ na faixa Norte, na zona de planícies, ao longo do Rio Rovuma.

A região de Unango é constituída por formações do pré-câmbrico primário. Os solos que se encontram na zona de Unango são superficiais (Litosolos), solos jovens (Cambisolos), solos vermelhos ferralíticos profundos e solos cinzentos profundos (MATE et al., 1985), bem drenados, destacam-se pela elevada fertilidade e grande potencial agrícola constituindo, assim, a zona Agroecológica 10 (MAE, 2005).

Conforme as variações topográficas e fisiográficas, a estrutura e composição do Miombo é modificada pelo clima, solo e altitude, distinguindo-se duma região para outra. O posto administrativo de Unango está inserido no distrito de Sanga que se encontra na região Zambesíaca, com subdivisão de Miombo 
decíduo seco que ocupa maior parte do distrito, na zona Norte e Nordeste e ao longo do Rio Rovuma e Miombo decíduo tardio das zonas planálticas e montanhosas do sul do distrito dominado por espécies do gênero Brachystegia (Brachystegia boehmii, B. spiciformis, vulgarmente conhecidas por messassas) e as espécies do gênero Uapaca (Massuco) nas zonas planálticas e montanhosas da Cordilheira de Sanga (MAE, 2005).

\section{Coleta de dados}

Para a coleta de dados inicialmente, buscou-se informação junto à população local a respeito do uso da vegetação e áreas de cultivo, para em seguida serem selecionados os ambientes objetos do estudo. As unidades amostrais foram constituídas de parcelas medindo $20 \mathrm{~m} \times 50 \mathrm{~m}$, sendo 15 parcelas nas áreas não antropizadas e outras 15 nas áreas abandonadas após agricultura, distribuídas aleatoriamente.

Todos os indivíduos arbóreos presentes nas parcelas das áreas não antropizadas com circunferência à altura da Peito (CAP) maior ou igual a $15.708 \mathrm{~cm}$, que foram consideradas adultas, foram mensurados $\mathrm{e}$ identificados. Como o rebrotamento geralmente ocorre no topo dos troncos cortados e, portanto, a altura da mensuração incluiu os troncos antigos (principalmente cortados em torno da altura do peito ou abaixo), para tal todos indivíduos com db maior ou igual a $5 \mathrm{~cm}(\mathrm{db}=$ diâmetro a $30 \mathrm{~cm}$ do nível do solo), também foram mensurados e identificados.

A identificação botânica dos indivíduos foi feita em campo pelo nome comum com ajuda de guias locais, e para os nomes científicos foram utilizadas as bibliografias comumente usadas para identificação de espécies vegetais nas savanas de Miombo da África Austral (PALGRAVE et al., 2002; VAN WYK et al., 2011).

Para os indivíduos que não foi possível identificar em campo foram coletados em sacos de coletas e numerados os materiais com fita crepe e que posteriormente eram prensados por meio dos procedimentos usuais de herborização e após secos identificava-se cada um deles com material disponível na Universidade Lúrio bem como consultas a especialistas. A grafia e a validade dos nomes científicos das espécies foram conferidas e atualizadas em revisões taxonômicas no Site da International plant names Index ${ }^{1}$, a nomenclatura das espécies segue a proposta da Angiosperm Philogeny Group (APG, 2009).

\section{Análise de dados}

\section{Suficiência amostral}

A suficiência amostral foi avaliada através de curvas de rarefação (GOTELLI et al., 2001) construídas com dados de abundância de espécies observadas nas 15 parcelas de amostragem para as áreas não antropizadas do Miombo nas áreas abandonadas após agricultura, através do programa EstimateS (COLWELL, 2013). As curvas de rarefação têm por objetivo deduzir qual teria sido a riqueza de espécies de uma comunidade se o esforço amostral tivesse sido reduzido a uma quantidade específica (MAGURRAN,

\footnotetext{
${ }^{1}$ http://www.ipni.org/ipni/plantnamesearchpage.do 
1988). A curva de acumulação de espécies foi calculada pela curva de rarefação ordenada pelo número de amostras.

\section{Parâmetros fitossociológicos}

Para o cálculo dos parâmetros fitossociológicos a CAP (circunferência a altura do peito) foi transformada em DAP (Diâmetro a altura do peito em $\mathrm{cm}$ ) por meio da divisão do valor de CAP pela constante $\pi=3.141593$ resultando em DAP mínimo de $5 \mathrm{~cm}$. Os parâmetros fitossociológicos para as espécies das áreas estudadas foram analisados através dos descritores fitossociológicos como: abundância (absoluta e relativa), dominância (absoluta e relativa) a frequência (absoluta e relativa), e índice de valor de importância (IVI) (Mueller-Dombois et al., 1974), as expressões são descritas a seguir:

$$
\begin{array}{r}
\mathrm{AB}_{\mathrm{abs}}=\frac{\mathrm{n}}{\mathrm{ha}} \quad \begin{array}{r}
\mathrm{AB}_{\text {rel }}=\frac{\mathrm{n} / \mathrm{ha}}{\mathrm{N} / \mathrm{ha}} \times 100 \\
\text { Onde: } \\
\mathrm{AB} \text { abs }=\text { Abundância absoluta; } \\
\mathrm{AB} \text { rel }=\text { Abundância relativa; }
\end{array} \\
\frac{\mathrm{n}}{\mathrm{ha}}=\text { Número de árvores de cada espécie por hectare } \\
\frac{\mathrm{N}}{\mathrm{ha}}=\text { Número total de árvores por hectare. }
\end{array}
$$

O cálculo da frequência, que mede a distribuição de cada espécie, em valores absoluto e relativo, sobre a área será obtido por meio das seguintes expressões:

$$
\mathrm{FR}_{\mathrm{abs}}=\frac{\mathrm{pi}}{P t} \times 100 \quad \mathrm{FR}_{\text {rel }}=\frac{\mathrm{FR}_{\mathrm{abs}}}{\sum_{\mathrm{i}=1}^{\mathrm{n}} \mathrm{FR}_{\mathrm{abs}}} \times 100
$$

Onde:

A dominância absoluta e a dominância relativa serão obtidas das seguintes formas:

$$
\mathrm{D}_{\mathrm{abs}}=\frac{\sum_{\mathrm{i}=1}^{\mathrm{n}} \mathrm{gi}}{\mathrm{ha}}=\frac{g}{h a} \quad \mathrm{D}_{\mathrm{rel}}=\frac{\sum_{i=1} \frac{g i}{h a}}{\sum \frac{g_{\mathrm{t}}}{h a}} \times 100=\frac{g / h a}{G / h a} * 100
$$

Onde:

$D_{a b s}=$ dominância absoluta, em $\mathrm{m}^{2}$, por hectare; $D_{\text {rel }}=$ dominância relativa (\%) espécie;

$\sum \mathrm{g}_{\mathrm{i}}=$ somatório das secções transversais do tronco das árvores; $\mathrm{g}=$ área basal por hectare; $\mathrm{G}=$ área basal total; ha = área amostrada, em hectare.

O índice de valor de importância (IVI) é estimado, por espécie, pela soma dos valores relativos da dominância, densidade e da frequência. 


\section{Ordenação e similaridade na composição e estrutura da vegetação}

Para investigar padrões de variação florístico entre as áreas foi utilizado o escalonamento multidimensional não métrico (NMDS: nonmetric multidimensional scaling), que é um método baseado em uma matriz de distância calculada por uma medida de distância ou de similaridade, em que o algoritmo busca localizar os pontos dos dados em duas ou mais dimensões (LEGENDRE et al., 1998). Para ordenação foram consideradas as parcelas, utilizando a medida de distância de Bray-Curtis, com dados transformados de abundância. A adequabilidade do gráfico de ordenação foi medida pelo valor do Stress em que valores de Stress inferiores a 0,05 representam um ajustamento perfeito entre as distâncias reais e as distâncias nos eixos, superiores a 0,20 são críticos, devendo ser usada alguma cautela na interpretação dos resultados, enquanto os valores superiores a 0,30 não podem ser interpretados (CLARKE, 1993). As análises fitossociológicas foram feitas através dos pacotes PREPARE e PARAMS do programa FITOPAC 2.1.2.85 (SHEPHERD, 2010) e as ordenações pelo NMDS foram feitas pelo programa estatístico R, usando o pacote Vegan (OKSANEN et al., 2012).

\section{RESULTADOS}

\section{Análise Florística dos Ambientes Estudados}

Neste estudo de levantamento florístico e fitosociológico realizado em dois ambientes de Miombo (preservado e antropizado), foram encontrados 965 indivíduos, eles foram considerados como pertencentes aos estratos arbóreo e arbustivo. A tabela 1 apresenta um resumo dos dados coletados.

Tabela 1: Resumo dos dados levantados no distrito de sanga, posto administrativo de Unango em dois ambientes.

\begin{tabular}{|l|l|l|l|l|}
\hline Ambiente & $\mathrm{N}^{\circ}$ de Parcelas & Área total amostrada (ha) & $\mathrm{N}^{\circ}$ de indivíduos encontrados & $\mathrm{N}^{\circ}$ de indivíduos por hectare \\
\hline $\mathrm{I}$ & 15 & 0,75 & 739 & 985,33 \\
\hline $\mathrm{II}$ & 15 & 0,75 & 226 & 150,67 \\
\hline
\end{tabular}

O ambiente I refere-se às áreas de Miombo em melhor estado de conservação e apresenta maior número de indivíduos pertencentes às categorias arbórea e arbustiva. O Ambiente II refere-se às áreas de Miombo mais antropizadas e possui menor número de indivíduos, em decorrência da degradação função da sua utilização pelas comunidades locais.

\section{Diversidade florística}

Observa-se que o Miombo mais conservado (Ambiente I) apresenta maior diversidade do que o Miombo antropizado (Ambiente II), uma vez que na primeira foram amostradas 21 famílias e 40 espécies botânicas (Tabela 2), enquanto que no ambiente II (antropizado), foram registradas apenas 10 famílias e 19 espécies, o padrão encontrado no presente estudo mostra que as curvas de acumulação de espécies (Figura 2) também foram maiores para áreas de Miombo não antropizado quando comparada com o outro sistema de uso de terra (áreas abandonadas após agricultura) e com base nessas curvas a amostragem foi suficiente para representar a composição florística das áreas de Miombo amostradas. 
Tabela 2: Demonstrativo dos números de famílias e espécies ocorrentes nos dois ambientes inventariados.

\begin{tabular}{|l|l|l|}
\hline & Ambiente I & Ambiente II \\
\hline Famílias & 21 & 10 \\
\hline Espécies & 40 & 19 \\
\hline
\end{tabular}

Curvas de rarefação

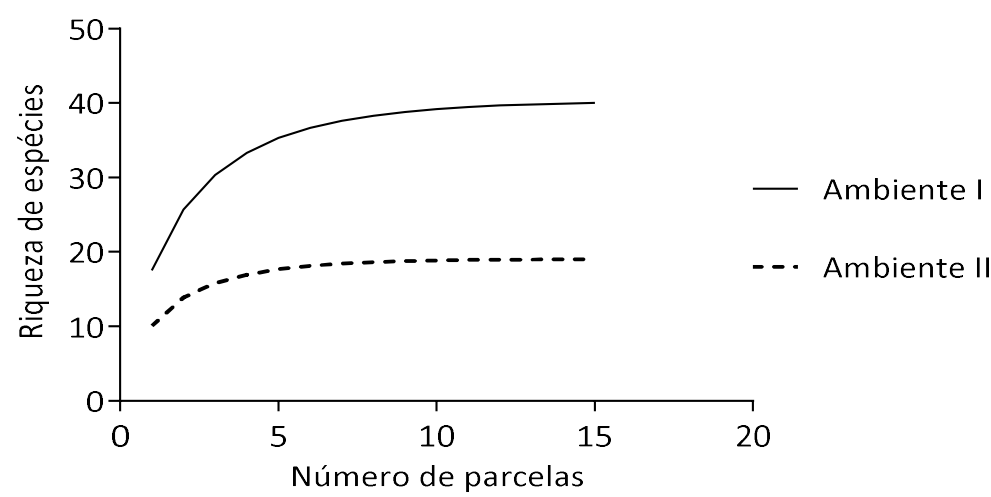

Figura 2: Curvas de acumulação de espécies para os dois ambientes estudados.

Na Tabela 3 observa-se que as famílias Fabaceae e Euphorbiaceae apresentam-se com maior número de indivíduos nos dois ambientes. As famílias Rubiaceae, Flacourtiaceae, Sterculiaceae, Annonaceae, Ochnaceae, Sapotaceae, Ebenaceae, Celastraceae, Capparaceae, Apiaceae, e Balanitaceae foram encontradas apenas no ambiente I (preservado), o que se deve possivelmente à antropização do ambiente II.

Tabela 3: Famílias e respectivas frequências de indivíduos encontradas em dois ambientes.

\begin{tabular}{|l|l|l|l|l|l|l|}
\hline \multirow{2}{*}{ Famílias } & Ambiente I & \multicolumn{3}{l|}{ Ambiente II } \\
\cline { 2 - 7 } & NInd & NSpp & \%Spp & NInd & NSpp & \%Spp \\
\hline Fabaceae & 365 & 14 & 35 & 101 & 8 & 42,11 \\
\hline Euphorbiaceae & 82 & 3 & 7,5 & 30 & 2 & 10,53 \\
\hline Combretaceae & 39 & 4 & 10 & 27 & 2 & 10,53 \\
\hline Chrysobalanaceae & 39 & 1 & 2,5 & 17 & 1 & 5,26 \\
\hline Apocynaceae & 47 & 1 & 2,5 & 20 & 1 & 5,26 \\
\hline Araliaceae & 36 & 1 & 2,5 & 2 & 1 & 5,26 \\
\hline Verbenaceae & 21 & 2 & 5 & 2 & 1 & 5,26 \\
\hline Rubiaceae & 21 & 1 & 2,5 & --- & --- & --- \\
\hline Loganaceae & 12 & 1 & 2,5 & 8 & 1 & 5,26 \\
\hline Flacourtiaceae & 12 & 1 & 2,5 & --- & --- & -- \\
\hline Myrtaceae & 9 & 1 & 2,5 & 12 & 1 & 5,26 \\
\hline Annonaceae & 6 & 1 & 2,5 & --- & --- & --- \\
\hline Polygalaceae & 6 & 1 & 2,5 & 7 & 1 & 5,26 \\
\hline Anacardiaceae & 7 & 1 & 2,5 & --- & --- & -- \\
\hline Ochnaceae & 6 & 1 & 2,5 & --- & --- & -- \\
\hline Sapotaceae & 7 & 1 & 2,5 & --- & --- & -- \\
\hline Ebenaceae & 9 & 1 & 2,5 & --- & --- & - \\
\hline Celastraceae & 5 & 1 & 2,5 & --- & --- & - \\
\hline Capparaceae & 6 & 1 & 2,5 & --- & --- & - \\
\hline Apiaceae & 3 & 1 & 2,5 & --- & --- & - \\
\hline Balanitaceae & 1 & 1 & 2,5 & --- & --- & - \\
\hline
\end{tabular}

Onde: $\mathrm{NInd}=$ número de indivíduos; NSpp= número de espécies; \%Spp= percentagem do número de espécies.

\section{Parâmetros Fitossociológicos}

\section{Estrutura florística horizontal}


Os parâmetros estudados foram frequência, densidade, dominância, índice de valor de importância, para as espécies do estrato arbustivo-arbóreo, cujos valores estão apresentados nas Tabelas 4 e 5.

Tabela 4: Número de indivíduos (NInd), frequência relativa (ReFr), dominância relativa (RelDo), densidade relativa (ReIDR), índice de valor de importância (IVI) das espécies ocorrentes no ambiente I.

\begin{tabular}{|c|c|c|c|c|c|}
\hline \multirow[t]{2}{*}{ Espécies } & \multicolumn{5}{|c|}{ Ambiente I } \\
\hline & NInd & RelDe & RelFr & RelDo & IVI \\
\hline Brachystegia spiciformis & 86 & 11,64 & 5,47 & 13,2 & 30,31 \\
\hline Julbernardia globiflora & 43 & 5,82 & 4,69 & 7,79 & 18,3 \\
\hline Uapaca kirkiana & 44 & 5,95 & 4,3 & 6,2 & 16,45 \\
\hline Diplorhynchus condylocarpon & 47 & 6,36 & 4,3 & 5,3 & 15,96 \\
\hline Parinari curatellifolia & 39 & 5,28 & 4,69 & 5,77 & 15,74 \\
\hline Cussonia arborea & 36 & 4,87 & 3,13 & 7,55 & 15,54 \\
\hline Brachystegia boehmii & 38 & 5,14 & 3,91 & 5,65 & 14,7 \\
\hline Pseudolachnostylis maprouneifolia & 26 & 3,52 & 3,52 & 6,28 & 13,31 \\
\hline Pericopsis angolensis & 34 & 4,6 & 4,3 & 3,86 & 12,75 \\
\hline Pterocarpus angolensis & 33 & 4,47 & 3,52 & 3,59 & 11,57 \\
\hline Burkea africana & 28 & 3,79 & 3,91 & 3,81 & 11,5 \\
\hline Brachystegia utilis & 30 & 4,06 & 3,13 & 2,74 & 9,92 \\
\hline Piliostigma thonningii & 20 & 2,71 & 3,13 & 2,62 & 8,45 \\
\hline Vitex doniana & 17 & 2,3 & 3,52 & 1,88 & 7,69 \\
\hline Catunaregam spinosa & 21 & 2,84 & 2,73 & 0,79 & 6,37 \\
\hline Uapaca nitida & 12 & 1,62 & 3,13 & 1,42 & 6,17 \\
\hline Combretum imberbe & 11 & 1,49 & 2,34 & 2,12 & 5,96 \\
\hline Cordyla africana & 13 & 1,76 & 1,95 & 2,1 & 5,81 \\
\hline Swartzia madagascariensis & 11 & 1,49 & 2,34 & 1,47 & 5,3 \\
\hline Terminalia stenostachya & 13 & 1,76 & 2,34 & 1,08 & 5,19 \\
\hline Terminalia sericea & 10 & 1,35 & 2,73 & 1,09 & 5,18 \\
\hline Strychnos spinosa & 12 & 1,62 & 1,56 & 1,25 & 4,43 \\
\hline Lonchocarpus bussei & 6 & 0,81 & 2,34 & 1,1 & 4,26 \\
\hline Syzygium guineense & 9 & 1,22 & 1,56 & 1,29 & 4,07 \\
\hline Flacourtia indica & 12 & 1,62 & 1,95 & 0,43 & 4,01 \\
\hline Dichrostachys cinerea & 9 & 1,22 & 1,95 & 0,69 & 3,86 \\
\hline Bauhinia petersiana & 8 & 1,08 & 1,56 & 1,15 & 3,79 \\
\hline Annona senegalensis & 6 & 0,81 & 1,95 & 0,83 & 3,6 \\
\hline Securidaca longepedunculata & 6 & 0,81 & 1,95 & 0,59 & 3,36 \\
\hline Dalbergia melanoxylon & 6 & 0,81 & 1,56 & 0,88 & 3,26 \\
\hline Ozoroa obovata & 7 & 0,95 & 1,95 & 0,35 & 3,25 \\
\hline Ochna schwerifurthiana & 6 & 0,81 & 1,56 & 0,82 & 3,19 \\
\hline Manilkara discolor & 7 & 0,95 & 0,78 & 1,15 & 2,88 \\
\hline Diospyros mespiliformis & 9 & 1,22 & 0,78 & 0,74 & 2,74 \\
\hline Combretum zeyheri & 5 & 0,68 & 1,17 & 0,75 & 2,6 \\
\hline Gymnosporia mossambicensis & 5 & 0,68 & 1,17 & 0,52 & 2,37 \\
\hline Vitex payos & 4 & 0,54 & 1,17 & 0,45 & 2,16 \\
\hline Boscia salicifolia & 6 & 0,81 & 0,78 & 0,53 & 2,13 \\
\hline Steganotaenia araliacea & 3 & 0,41 & 0,78 & 0,14 & 1,33 \\
\hline Balanites muahagamii & 1 & 0,14 & 0,39 & 0,02 & 0,55 \\
\hline Total & 739 & 100 & 100 & 100 & 300 \\
\hline
\end{tabular}

As espécies mais comuns ocorrentes nos dois ambientes encontram-se bem distribuídas espacialmente, embora estes ambientes não sejam tão homogêneos (Tabelas 4 e 5). Todavia, as diferenças entre os dois ambientes são marcantes: no ambiente I, a espécie de maior importância ecológica é Brachystegia spiciformis (IVI = 30,31), seguida de Julbernardia globiflora (IVI = 18,3); Uapaca kirkiana (IVI = 16,45); Diplorhynchus condylocarpon (IVI =15,96); Parinari curatellifolia (IVI =15,74); Cussonia arborea 
(IVI=15,54); Brachystegia boehmii (IVI=14,7); Pseudolachnostylis maprouneifolia (IVI=13,31); Pericopsis angolensis (IVI=12,75); Pterocarpus angolensis (IVI=11,57).

No ambiente II sobressai-se Uapaca kirkiana (IVI = 30,38) seguida de Diplorhynchus condylocarpon (IVI = 28,19); Pterocarpus angolensis (IVI = 27,87); Brachystegia boehmii (IVI = 23,35); Parinari curatellifolia (IVI =23,34); Syzygium guineense (IVI =19,9); Dichrostachys cinerea (IVI = 19,76); Combretum zeyheri (IVI=18,99); Terminalia sericea (IVI=18,34), e Pericopsis angolensis $(\mathrm{IVI}=15,98)$. A composição florística do Miombo variou nos diferentes estágios de sucessão.

Tabela 5: Número de indivíduos (NInd), frequência relativa (ReFr), dominância relativa (RelDo), densidade relativa (ReIDR), índice de valor de importância (IVI) das espécies ocorrentes no ambiente II.

\begin{tabular}{|c|c|c|c|c|c|}
\hline \multirow[t]{2}{*}{ Espécies } & \multicolumn{5}{|c|}{ Ambiente II } \\
\hline & NInd & RelDe & RelFr & RelDo & IVI \\
\hline Uapaca kirkiana & 25 & 11,06 & 9,42 & 9,89 & 30,38 \\
\hline Diplorhynchus condylocarpon & 20 & 8,85 & 9,42 & 9,92 & 28,19 \\
\hline Pterocarpus angolensis & 23 & 10,18 & 8,7 & 9 & 27,87 \\
\hline Brachystegia boehmii & 19 & 8,41 & 7,97 & 6,97 & 23,35 \\
\hline Parinari curatellifolia & 17 & 7,52 & 8,7 & 7,13 & 23,34 \\
\hline Syzygium guineense & 12 & 5,31 & 5,07 & 9,52 & 19,9 \\
\hline Dichrostachys cinerea & 16 & 7,08 & 6,52 & 6,16 & 19,76 \\
\hline Combretum zeyheri & 14 & 6,19 & 6,52 & 6,27 & 18,99 \\
\hline Terminalia sericea & 13 & 5,75 & 5,8 & 6,79 & 18,34 \\
\hline Pericopsis angolensis & 13 & 5,75 & 2,9 & 7,33 & 15,98 \\
\hline Brachystegia spiciformis & 10 & 4,42 & 5,8 & 3,79 & 14,01 \\
\hline Piliostigma thonningii & 8 & 3,54 & 4,35 & 3,07 & 10,95 \\
\hline Strychnos spinosa & 8 & 3,54 & 3,62 & 2,63 & 9,79 \\
\hline Swartzia madagascariensis & 6 & 2,65 & 3,62 & 2,58 & 8,85 \\
\hline Pseudolachnostylis maprouneifolia & 5 & 2,21 & 3,62 & 2,73 & 8,56 \\
\hline Securidaca longepedunculata & 7 & 3,1 & 2,17 & 2,81 & 8,08 \\
\hline Julbernardia globiflora & 6 & 2,65 & 2,9 & 2,39 & 7,94 \\
\hline Vitex doniana & 2 & 0,88 & 1,45 & 0,57 & 2,9 \\
\hline Cussonia arborea & 2 & 0,88 & 1,45 & 0,47 & 2,8 \\
\hline Total & 226 & 100 & 100 & 100 & 300 \\
\hline
\end{tabular}

\section{Similaridade e ordenação na composição e estrutura entre as áreas}

A análise de ordenação apresentou uma separação entre os dois ambientes estudados, mostrando as áreas abandonadas após agricultura (Ambiente II) são mais distintas em termos de composição de espécies em relação ao Miombo conservado (Ambiente I) que existe uma diferença na composição de espécies entre os ambientes.

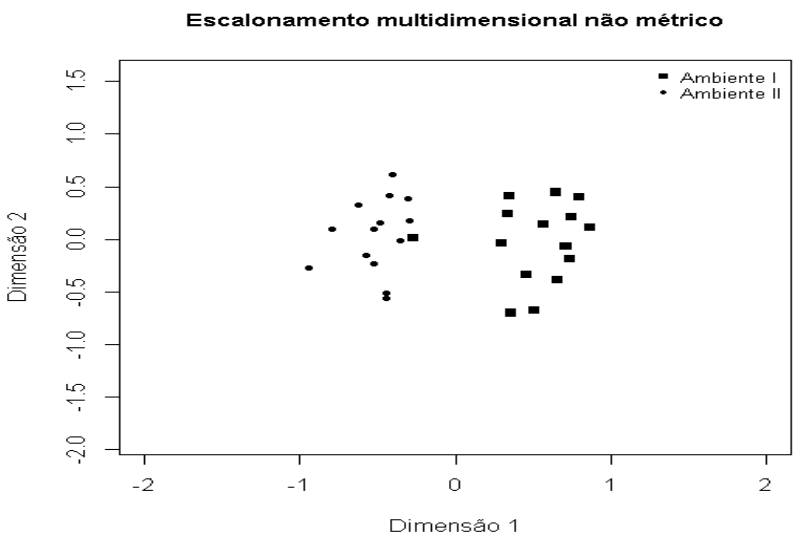

Figura 3: Diagrama de ordenação pelo escalonamento multidimensional não métrico com dados de presença-ausência para os ambientes estudados. 


\section{DISCUSSÃO}

Observou-se no presente estudo baixa riqueza de espécies nos ambientes antropizados em relação ao ambientes não antropizados e esse resultado é esperado, já que ambientes não alterados tendem a ser mais complexos e ricos do que áreas alteradas (OKIROR et al., 2012; KANUNGWE et al., 2013; GONÇALVES et al., 2017). O destaque para família Fabaceae é esperado, uma vez que é frequentemente registrada entre as mais importantes na Savanas do Miombo (TUITE et al., 1990; ZIMUDZI et al., 2013; MWAKALUKWA et al., 2014; JEW et al., 2016; GONSALVES et al., 2017). Esta família constitui cerca de 35\% de todas as árvores mensuradas na área de Miombo conservado, 42,11\% nas áreas abandonadas após agricultura.

As savanas de Miombo seco são historicamente definidas por espécies da Família Fabaceae, principalmente do gênero Brachystegia e Julbernardia (TIMBERLAKE et al., 2011). E o presente estudo nas áreas de Miombo conservado e nas áreas abandonadas após agricultura confirmou a dominância da família em termos de gêneros e números de espécies. Além da família Fabaceae, as famílias Euphorbiaceae e Combretaceae apresentaram-se com maior riqueza de espécies nessa formação florestal.

As espécies mais importantes observadas nas áreas do ambiente I no presente estudo são os comumente encontradas nas áreas de Miombo em Moçambique (RIBEIRO et al., 2008; HOFIÇO et al., 2015). Em Moçambique os gêneros Brachystegia, Julbernardia geralmente estão associadas as espécies como Diplorhynchus condylocarpon, Parinari curatellifolia e Pseudolachnostylis maprouneifolia. A espécie Brachystegia spiciformis foi a espécie sociologicamente importante na área de Miombo conservado (Ambiente I) e segue o padrão típico para estas formações florestais e está associada a capacidade dela de competir com indivíduos de outras espécies em solos porosos e de baixa fertilidade.

Nas áreas de abandonadas após agricultura, observamos a dominância de espécies tolerantes ao fogo, como por exemplo Pterocarpus angolensis e Diplorhynchus condylocarpon (TRAPNELL, 1959; LAWTON, 1978), e por espécies pouco resistentes ao fogo, como por exemplo S. guineense e U. kirkiana (KANUNGWE et al., 2013). Peters (1994), Stromgaard (1984), e Kanungwe et al., (2013) reportaram a dominância de espécies tolerantes a seca e ao fogo nas áreas abandonadas após agricultura de corte-e-queima, já que o fogo é importante na quebra da dormência das sementes, facilitando a germinação e rebrotação de algumas espécies (Stromgaard, 1984). Este fato pode explicar a prevalência nas áreas de espécies $P$. angolensis e $D$. condylocarpon. Pterocarpus angolensis por exemplo é uma espécie intolerante à sombra e seu crescimento e bom desempenho está associado a áreas abertas e com máxima exposição a luz (GRAZ, 1996). Outro fator que pode favorecer a prevalência dessas espécies e que nas áreas em recuperação após agricultura há maiores probabilidades de incidências de incêndios (BOALER et al., 1966), devido a maior quantidade de acúmulo de combustíveis.

Um aspeto relevante e que foi observado nas áreas abandonadas após agricultura é a presença de espécies da família Combretaceae (p. ex., Combretum zeyheri e Terminalia sericea). A ocorrência de espécies de Combretum após perturbação também foi relatada em outros estudos sobre a sucessão secundária do Miombo no sul e Centro da África (STROMGAARD, 1987; BACKÉUS et al., 2006; GONSALVES et al., 2017). A 
presença de $C$. zeyheri sugere a alta pressão de uso de terra, já que a espécie tem crescimento rápido e dominante em áreas mais abertas e alteradas, como os estágios iniciais de sucessão (JEW et al., 2016). As observações nas florestas secundárias permitem sugerir que dentro de certas regiões são dominadas pelo pequeno grupo de espécies pioneiras (FINEGAN, 1992).

A baixa similaridade em termos de composição de espécies entre as áreas abandonadas após agricultura e do Miombo conservado pode ser atribuída a atividades antropogênicas realizadas nessas áreas. Essas atividades incluem o desmatamento de terras para cultivo com a prática de agricultura de corte-equeima que causa o desaparecimento de espécies lenhosas (AGEA et al., 2007).

As áreas abandonadas após agricultura mostram que este tipo de uso de terra tem um grande impacto na composição e estrutura do Miombo, principalmente porque o cultivo pode resultar na mortalidade de algumas plantas devido as injurias causadas durante o cultivo (STRANG, 1974). A remoção de vegetação para o cultivo resulta por vezes na retirada de mudas ou espécies em regeneração, pois são percebidas como ervas daninhas pelos agricultores. Além disso, o uso do fogo prejudica a germinação das sementes de algumas espécies presentes no banco de sementes do solo, possivelmente por estas não possuírem capacidade de germinar após passagem dele.

\section{CONCLUSÕES}

As áreas de Miombo sem antropização apresentam um melhor estado de conservação e consequentemente uma maior diversidade, tanto em número de famílias, quanto em número de espécies identificadas. A família Fabaceae é a mais rica, as espécies sociologicamente importantes são as mais dominantes em Savanas de Miombo Brachystegia e Julbernardia associadas a outras espécies como Diplorhynchus condylocarpon, Parinari curatellifolia e Pseudolachnostylis maprouneifolia. As espécies dominantes nas áreas perturbadas são as comumente mais encontradas nesses ambientes e são capazes de crescer em áreas antropizadas. A diminuição da diversidade e da cobertura vegetal tem se dado provavelmente pela ação antrópica dos moradores no local do estudo.

\section{REFERÊNCIAS}

AGEA, J. G.; OBUA, J.; KABOGGOZA, J. R. S.; WAISWA, D.. Diversity of indigenous fruit trees in the traditional cottonmillet farming system: The case of Adwari subcounty, Lira district, Uganda. African Journal of Ecology, v.45, n.3, p.3943, 2007.

APG III. The Angiosperm Phylogeny Group III. An update of the Angiosperm Phylogeny Group classification for the orders and families of higher plants: APG III. Botanical Journal of the Linnean Society, London, v.161, n.2, p.105$121,2009$.

BACKÉUS, I.; PETTERSSON, B.; STRÖMQUIST, L.; RUFFO, C.. Tree communities and structural dynamics in miombo (Brachystegia-Julbernardia) woodland, Tanzania. Forest Ecology and Management, v.230, n.1-3, p.171-178, 2006.
BOALER, S. B.; SCIWALE, K.C. ECOLOGY OF A MIOMBO SITE, LUPA NORTH FOREST RESERVE, TANZANIA II. Plant Communities and Seasonal Variation in the Vegetation Author(s): Journal of Ecology, v.54, n.2, p.465-479, 1966.

CLARKE, K. R.. Non-parametric multivariate analyses of changes in community structure. Australian Journal of Ecology, v.18, n.1988, p.117-143, 1993. DOI: http://doi.org/10.1111/j.1442-9993.1993.tb00438.x

COLWELL, R. K.. EstimateS: Statistical estimation of species richness and shared species from samples. Software and User's Guide, Version 9 and earlier. 2013.

FINEGAN, B.. The Management Potential of Neotropical Secondary Lowland Rain Forest. Forest Ecology and Management, v.47, p.295-322, 1992. 
GONÇALVES, F. M. P.; REVERMANN, R.; GOMES, A. L.; AIDAR, M. P.; FINCKH, M.; JÜRGENS, N.. Tree species diversity and composition of Miombo woodlands in south- central Angola, a chronosequence of forest recovery after shifting cultivation. International Journal of Forestry Research, v.2017, p.13, 2017. DOI:

http://doi.org/doi:10.1155/2017/6202093

GOTELLI, N. J.; COLWELL, R.. Quantifying biodiversity: procedures and pitfalls in the measurment and comparison of species richness. Ecology Letters, v.4, p.379-391, 2001. DOI: http://doi.org/10.1046/j.1461-0248.2001.00230.x

GRAZ, F. P.. Management of a Pterocarpus angolensis under the influence of fire and land use. (Msc Dissertation) University of Stellenbosch, 1996.

HOFIÇO, N. S. A.; FLEIG, F. D.. Diversity and Structure of Miombo woodlands in Mozambique Using a Range of Sampling Sizes. Journal of Agricultural Science and, v.5, p.679-690, 2015. DOI: http://doi.org/10.17265/21616264/2015.10.005

JEW, E. K. K.; DOUGILL, A. J.; SALLU, S. M.; O'CONNELL, J.; BENTON, T. G.. Miombo woodland under threat: Consequences for tree diversity and carbon storage. Forest Ecology and Management, v.361, p.144-153, 2016. DOI: http://doi.org/https://doi.org/10.1016/j.foreco.2015.11.011

KANUNGWE, F.; HELEN, C.; JOHN, A.; VINYA, R.. Floristic composition, species diversity and carbon storage in charcoal and agriculture fallows and management implications in Miombo woodlands of Zambia. Forest Ecology and Management, v.304, p.99-109, 2013. DOI: http://doi.org/10.1016/j.foreco.2013.04.024

KARTHIK, T.; VEERASWAMI, G. G.. Review article Forest recovery following shifting cultivation : an overview of existing research. Tropical Conservation Science, v.2, n.4, p.374-387, 2009.

LANDRY, J.; CHIRWA, P. W.. Analysis of the potential socioeconomic impact of establishing plantation forestry on rural communities in Sanga district, Niassa province, Mozambique. Land Use Policy, v.28, n.3, p.542-551, 2011. DOI: http://doi.org/10.1016/j.landusepol.2010.11.001

LAWTON, R. M.. Study of dynamic ecology of Zambian vegetation. Journal of Ecology, v.66, p.175-200, 1978.

LEGENDRE, P.; LEGENDRE, L.. Numerical Ecology. 2 ed. Amsterdam: Elsevier, 1988.

MAGURRAN, A.E. Ecological diversity and its measurement. New Jersey: Princeton University Press. 179p. 1988.

MATE, A. O.; WAMBEKE, J. V.. Os solos da região de Unango: província de Niassa. Nota técnica n.30. Maputo: Série Terra e Água do Instituto Nacional de Investigação Agronómica, 1985.

MARACAJÁ, P. B.; HENRIQUE, C.; BATISTA, F.; SOUSA, A. H.. Levantamento florístico e fitosociológico do extrato arbustivo- arbóreo de dois ambientes na Vila Santa Catarina, Serra Do Mel/RN, 3. 2003.

MARZOLI, A.. Inventário Florestal Nacional-Relatório Final de Avaliação integrada das florestas de Moçambique. Maputo: Ministério da Agricultura, 2007.

MAE. Ministério da Administração Estatal. Perfil do distrito de Sanga, Província do Niassa. Série: Perfis dos distritos. Maputo: Ministério da Administração Estatal, 2005.

MOURANA, B.; SERRA, C. M.. 20 Passos para a Sustentabilidade Florestal em Moçambique. Edição: Amigos da Floresta/Centro de Integridade Pública. Maputo, 2007.

MUELLER-DOMBOIS, D.; ELLENBERG, H.. Aims and methods of vegetation ecology. New York: John Wiley \& Sons, 1974.

MWAKALUKWA, E. E.; MEILBY, H.; TREUE, T.. Floristic Composition, Structure, and Species Associations of Dry Miombo Woodland in Tanzania. 2014.

NHANTUMBO, I.; MACQUEEN, D.; CRUZ, R.; SERRA, A.. Investing in locally controlled forestry in Mozambique: Potential for promoting sustainable rural development in the province of Niassa. 2013.

OKIROR, P.; CHONO, J.; NYAMUKURU, A.; LWANGA, J. S.; SASIRA, P.; DIOGO, P.. Variation in Woody Species Abundance and Distribution in and around Kibale National Park, Uganda. ISRN Forestry, v.2012, p.1-9., 2012. DOI: http://doi.org/10.5402/2012/490461

OKSANEN, J.; BLANCHET, F. G.; KINDT, R.; LEGENDRE, P.; MINCHIN, P. R.; O'HARA, R. B.; SIMPSON, G. L.; SOLYMOS, P.; STEVENS, M. H. H.. Vegan: Community Ecology Package. 2012.

PALGRAVE, K. C.; DRUMMOND, R. B.; MOLL, E. J.; PALGRAVE, M. C.. Trees of southern Africa, 3 ed. Cape Town: Struik Publishers, 2002.

PETERS, C. M.. Sustainable Harvest of Non-timber Plant Resources in Tropical Moist Forest: An Ecological Primer. Washington, 1994.

RIBEIRO, N. S.; SHUGART, H. H.; WASHINGTON-ALLEN, R.. The effects of fire and elephants on species composition and structure of the Niassa Reserve, northern Mozambique. Forest Ecology and Management, v.255, n.5-6, p.1626-1636, 2008. DOI: http://doi.org/10.1016/j.foreco.2007.11.033

SHEPHERD, G. J.. FITOPAC: Versão 2.1. Campinas: Departamento de Botânica, Universidade Estadual de Campinas/UNICAMP, 2010.

SILESHI, G.; AKINNIFES, I. F. K.; AJAYI, O. C.; CHAKEREDZA, S.; KAONGA, M.; MATAKALA, P. W.. Contribution of agroforestry to ecosystem services in the Miombo ecoregion of eastern and southern Africa. African Journal Environment Science Technology, v.1, n.4, p.68-80, 2007.

STRANG, R. M.. Some man-made changes in successional trends on the Rhodesian Highveld. The Journal of Applied Ecology, v.11, n.1, p.249-263. 1974.

STROMGAARD, P.. The immediate effect of burning and ashfertilization. Plant and Soil, v.80, n.3, p.307-320, 1984. DOI: http://doi.org/10.1007/BF02140038 
STROMGAARD, P.. Early secondary succession on abandoned shifting cultivator's plots in the Miombo of South central Africa. Biotropica, v.18, p.97-106, 1987.

SYAMPUNGANI, S.; GELDENHUYS, C. J.; CHIRWA, P. W. Regeneration dynamics of miombo woodland in response to different anthropogenic disturbances : forest characterisation for sustainable management. Agroforestry Systems, v.90, n.4, p.563-576, 2015. DOI: http://doi.org/10.1007/s10457-015-9841-7

TIMBERLAKE, J.; CHIDUMAYO, E.. Miombo Ecoregion Vision report, Occasional publications in Biodiversity report no 20. Biodiversity foundation for Africa, Bulawayo, 2011.
TRAPNELL, C. G.. Ecological Results of Woodland and Burning Experiments in Northern Rhodisia. Journal of Ecology, v.47, p.129-168, 1959.

TUITE, P.; GARDINER, J. J.. The miombo woodlands of central, eastern and southern Africa. Irish Forestry, v.47, n.2, p.90-107, 1990.

VAN WYK, B.; VAN WYK, P.. Field guide to trees of Southern Africa. Cape Town: Struik Publishers, 2011.

ZIMUDZI, C.; MAPAURA, A.; CHAPANO, C.; DURI, W.. Woody species composition, structure and diversity of Mazowe Botanical Reserve, Zimbabwe. JBES, v.3, n.6, p.17-29, 2013.

A CBPC - Companhia Brasileira de Produção Científica (CNPJ: 11.221.422/0001-03) detém os direitos materiais desta publicação. Os direitos referem-se à publicação do trabalho em qualquer parte do mundo, incluindo os direitos às renovações, expansões e disseminações da contribuição, bem como outros direitos subsidiários. Todos os trabalhos publicados eletronicamente poderão posteriormente ser publicados em coletâneas impressas sob coordenação da Sustenere Publishing, da Companhia Brasileira de Produção Científica e seus parceiros autorizados. Os (as) autores (as) preservam os direitos autorais, mas não têm permissão para a publicação da contribuição em outro meio, impresso ou digital, em português ou em tradução. 